\title{
The experience of patients with bipolar disorder from diagnosis disclosure: A qualitative study
}

\author{
Azam Zolfi Kashani ${ }^{1}$, Hadi Ranjbar ${ }^{2}$, Maryam Rasoulian ${ }^{3}$, Amir Shabani $^{4}$, Mohammad Ghadirivasfi ${ }^{3}$, \\ Homa Mohammadsadeghi*3 (1)
}

Received: 16 Oct 2019

Published: 20 Apr 2020

\section{Abstract}

Background: Disclosure of the diagnosis is an essential part of the treatment process and an important part of patient rights. However, it can be a very stressful experience, especially in mental health disorders. Patients with bipolar disorder have a unique experience of receiving and managing their diagnosis. The objective of the current study was to explore the experience of patients with bipolar disorder of diagnosis disclosure.

Methods: This was a qualitative study. Participants were recruited from patients who knew their disorder's name using purposive sampling method. The inclusion criteria were being diagnosed by a psychiatrist as having bipolar disorder and the disclosure was conducted by a psychiatrist. Sixteen semi-structured, in-depth interviews were conducted with twelve patients. Data were analyzed using thematic content analysis.

Results: Patients received their diagnosis name in three steps including Wandering in Unknowns, Limited Brightness and Reaching to a Relative Insight. Patients believed that disclosure of the diagnosis was not accompanied by enough information.

Conclusion: The disclosure of diagnosis in patients with bipolar disorder without providing enough information is stressful and is not helpful in empowering these patients. Based on our results, disclosure of diagnosis to patients with bipolar disorder was not conducted with enough information and patients had problems for understanding their symptoms and treatments.

Keywords: Disclosure, Bipolar disorder, Mental health, Medical ethics, Patient rights

\author{
Conflicts of Interest: None declared \\ Funding: None \\ *This work has been published under CC BY-NC-SA 1.0 license. \\ Copyright $($ Iran University of Medical Sciences
}

Cite this article as: Zolfi Kashani A, Ranjbar H, Rasoulian M, Shabani A, Ghadirivasfi M, Mohammadsadeghi H. The experience of patients with bipolar disorder from diagnosis disclosure: A qualitative study. Med J Islam Repub Iran. 2020 (20 Apr);34:36. https://doi.org/10.47176/mjiri.34.36

\section{Introduction}

Bipolar disorder is one of the common psychiatric disorders with an episodic instability in the mood, behavior, and insight (1). It has a significant impact on individual performance, reduces the quality of life (2), and amplifies suicide

Corresponding author: Dr Homa Mohammadsadeghi, mohammadsadeghi.h@iums.ac.ir

1. Medical School, Iran University of Medical Sciences, Tehran, Iran

2. Mental Health Research Center, Psychosocial Health Research Institute, Iran University of Medical Science, Tehran, Iran

3. Mental Health Research Center, Tehran Institute of Psychiatry- School of Behavioral Sciences and Mental Health, Iran University of Medical Sciences, Tehran, Iran

4. Mental Health Research Center, Mood Disorders Research Group, Iran University of Medical Sciences, Tehran, Iran risk (3). Fortunately, with advances in the treatment of psychiatric illnesses, including bipolar disorder, many of these patients are able to live more productively (4). Despite these developments, it seems that an essential aspect of patient management that is related to their right to know their

\section{$\uparrow$ What is “already known” in this topic:}

Disclosure of diagnosis is a hard part of the treatment process especially in patients with end poor prognosis conditions like mental health illnesses. Patients with bipolar disorder usually do not receive their diagnosis name because it is believed that it is not helpful.

\section{$\rightarrow$ What this article adds:}

Our results showed that patients with bipolar disorder who do not know their disorder's name face several problems. With partial disclosure and not providing enough information, they may have more problems. Our results support full disclosure with complete information for patients with bipolar disorder. 
diagnosis did not get enough attention (5). Disclosure of diagnosis is an important part of the treatment process (6-8) which empowers the patients. Disclosure of diagnosis of patients with mental disorders is a controversial topic.

There are three different approaches regarding the disclosure of psychiatric diagnosis. The first approach is based on medical models and aims to reduce the symptoms of the disease. The second approach is the rehabilitation model that seeks to improve the performance of patients (9). The third approach is the recovery approach. The aim of recovery is "enabling a person with a mental health problem to live a meaningful life in a community of his or her choice while striving to achieve his or her full potential." (10) In order to move toward recovery, patients must know their diagnosis and receive sufficient information about it (9).

Many patients with bipolar disorder do not know the name of their illness (11). Studies have shown that in different countries, the approach to disclosing the diagnosis is different (12-14). In Iran, the disclosure approach is closer to medical models. Although therapeutic approaches have moved toward rehabilitation in recent years, the experience of researchers suggests that many patients with bipolar disorder do not know their own diagnosis. However, the disclosure of psychiatric diagnoses has both advocates and opponents. Both groups have ethical considerations regarding the disclosure of diagnosis. Advocates emphasize the importance of giving adequate information and decision-making power to patients. Opponents put emphasis on the possibility of trauma to the patient (15-17). At the same time, the patient's own experience of disclosing psychiatric diagnosis has been underestimated. This is while patients are the most important beneficiaries to disclose a diagnosis.

Patients with bipolar disorder may experience a lot of problems because they do not know their illness name. It is tough to get help when you do not know your disease name. Patients' behaviors are not justifiable in the two stages of depression and mania by the patients themselves and others (13). Getting information from sources like the internet is complicated. In such a situation, it's almost impossible to find support from support groups and associations (13). However, less attention has been paid to the patient's own experience of disclosing psychiatric diagnosis while they are the most critical beneficiaries in this subject.

Studies about the experience of patients with bipolar disorder from diagnosis disclosure and how they handle it are limited. On the other hand, the features of patients afflicted with bipolar disorder could be various in different regions and cultures (18).

Several studies have assessed psychiatrists' and therapists' opinions about diagnosis disclosure (14, 15, 18-20). Their results showed that there are different approaches to diagnosis disclosure in patients with mental illness. However, there were little known about the patient experience from receiving their diagnosis name and how they handle it. We did not find any study that assessed the patients' experience regarding this topic. The objective of the current study was to explore the experience of patients with bipolar disorder of diagnosis disclosure.

\section{Methods}

\section{Study design and participants}

This was a qualitative study. The participants were patients with bipolar disorder who knew the name of their disease. The diagnosis disclosure was done by psychiatrists. An announcement was made to invite patients who had inclusion criteria to participate in the study at the Iran Psychiatric Hospital, affiliated to Iran University of Medical Sciences. The inclusion criteria were being diagnosed by at least one psychiatrist as having bipolar disorder, they had the diagnosis for at least one year and it was confirmed, they were hospitalized in the Iran Psychiatric Hospital for bipolar disorder before, the disclosure of diagnosis was conducted by a psychiatrist at least one year ago, being 18 years old and above, willingness to participate in the study, not being in the psychotic, mania or depression phases. At first, each patient was examined by a psychiatrist to have clinical insight to participate in the study (True emotional insight based on clinical rating of insight. Twenty-seven patients volunteered to participate. Among them 8 participants had mood episodes. Seven patients had psychosis symptoms. The sampling started purposefully. Then, to reach the maximum variation of the study sample, the sampling continued by choosing patients from both sexes, with different age groups and educational levels.

\section{Data gathering}

The data was gathered using the semi-structured interview. The first and second author did the interviews. The first author is a psychiatric resident and the second author is a Ph.D. in Nursing. An interview guide was developed for first interviews by the research team. The interview guide had three main parts including initial questions such as "How did you find about your disease name?" The intermediate questions were the central part of the interview. It consists of questions such as "What was your reaction to your diagnosis?", "What information did you get from your doctor?" or "How did you find extra information?". The ending questions were the last part of the interview guide. Examples of ending questions were "Do you have anything to add?". New questions were raised from data analysis and were added to the guideline in the process of data gathering. The interviews were done in a comfortable place. Sixteen interviews with twelve patients the . Four participants accepted to be interviewed again. Interviews lasted between 43 to 72 minutes with an average of 51 minutes. Eight participants were men, and four participants were women. The demographic information of participants is presented in Table 1.

\section{Data analyzing}

Braun and Clarke (21) method of thematic analysis was used in the process of data analysis. Familiarizing with that data was the first step. The first author listened and transcribed all the interviews. Then she read them several times. The second step was generating initial codes. All research team participated in the coding process. The interviews were coded by the first author under the supervision of the second author in a file with Microsoft word format. Then they were emailed to all members of the team. The 
Table 1. Demographic characteristics of study participants

\begin{tabular}{lcccc}
\hline No & Gender & Age & Marital status & Education Level \\
\hline 1 & Male & 34 & Divorced & Diploma \\
2 & Male & 57 & Married & Bachelor Degree \\
3 & Male & 25 & Single & Diploma \\
4 & Female & 35 & Single & Associate Degree \\
5 & Female & 29 & Single & MSc \\
6 & Male & 35 & Married & Middle School degree \\
7 & Female & 20 & Married & Middle School degree \\
8 & Female & 46 & Married & Associate Degree \\
9 & Male & 37 & Single & Associate Degree \\
10 & Female & 35 & Divorced & Diploma \\
11 & Male & 28 & Single & MSc \\
12 & Female & 35 & Single & Bachelor Degree \\
\hline
\end{tabular}

coded segments were reviewed by all members of the research team. Each participant was contacted after the interview was coded. They were asked to participate in another meeting, if possible. The codes were checked with the participants who accepted to participate again. Searching for themes was the third step. From the first interview, related coded were grouped to form primary categories. As interviews progressed, categories and sub-categories became more specific and more advanced. In this step, the researcher searched for patterns in data to form themes by connecting relevant categories to each other. The primary themes were identified from the patterns in the data. The fifth step was choosing the main theme(s). The main theme was chosen after reviewing primary themes. The selected theme was named in the sixth step. Sampling was stopped after reaching data saturation. The saturation of the data was determined after completing the characteristics of all themes and sub-themes.

\section{Ethical Consideration}

This study was approved by the Ethics Committee of Iran University of Medical Sciences (IR.IUMS.FMD. REC1396.9311286005). Informed written consent was obtained from all participants. All data were managed and analyzed anonymously. Member check and peer check was conducted to increase credibility. All the procedures were described in order to increase the transferability.

\section{Results}

Twelve patients were interviewed, including eight men and four women. The age range of participants was 20 to
57 years. The characteristics of the study participants are presented in Table 1.

Our results showed that patients who received their diagnosis name from their therapists went through a route named "wandering in unknowns to reaching a relative insight" which we consider as the main theme. We presented this theme in a three-step process. The steps which were our subthemes were "wandering in unknowns", "limited brightness", and "reaching a relative insight". The main theme, subthemes, and codes of bipolar patients' experience regarding disclosure of their diagnosis is presented in Table 2.

\section{Wandering in unknowns}

The first sub theme was "wandering in unknowns". At the onset of the disorder, our participants experienced symptoms that were new and strange to them. In the mania phase, patients experienced delusions such as grandiosity. They may feel that they have supernatural powers. They may do things that are strange for themselves and others. At this stage, patients are wandering among strange emotions and weird thoughts. Patients and their families do not find reasonable grounds for patients' thoughts and behaviors. In the depression phase, the mood of the patient decreases significantly. The patient may have feeling like being energetic, lack of energy and hope. Patients may experience suicidal thoughts. The question of the patient and the family at this stage is "why these things happen".

"I had strange thoughts and feelings, those two days. I felt that I had supernatural powers. I believed that I am like one of the prophets of God. Now that I'm talking about it, I think how ridiculous were those thoughts. But they were

Table 2. Themes, subtheme, and codes of bipolar patients' experience regarding disclosure of their diagnosis

\begin{tabular}{lc}
\hline Main Theme & Subthemes \\
\hline Wandering in unknowns to & Wandering in unknowns \\
reaching to a relative insight &
\end{tabular}

Limited brightness

Reaching a relative insight

\section{Codes}

Experiencing new things

Encountering with unknown experiences

Thoughts and beliefs are strange for patients and significant others

The patient is accused of substance abuse

Being treated without disclosure of diagnosis

Finding out something is wrong

Asking from the therapist

Disclosure of the diagnosis without giving enough information Experiencing stress

Coming out of the darkness of the unknowns

The inadequacy of information in the education by therapists

Seeking information from other sources

Failure to make important decisions based on the received information Relative awareness 
real to me at the time". (Participant 6)

"I questioned myself and my family some questions. What is wrong with you? Why do you do such things? For example, my financial situation was not good, but I lent money to my friend". (Participant 9)

"Sometimes, I thought I should die, and the world was worthless, while I was very energetic before. My family was always worried about hurting myself. Once I fell from the roof of a building on a construction site. I believed I could walk on air. My family thought it was a suicide". (Participant 2)

Limited brightness: The second sub theme was "limited brightness". In many cases, patients with bipolar disorder are treated without knowing their diagnostic name. These patients do not have enough insight when they are treated, or the psychiatrist does not disclose the diagnosis to them. The patients we interviewed knew the name of their illness. The diagnosis was disclosed by their psychiatrists. According to patient experience, the name of the diagnosis was disclosed to them when they asked their illness name. They did not receive much information about their disorder. By knowing the name of the disorder, some of the patient's headache had been resolved. By knowing the name of the disorder, some of the wanderings of the patients were disappeared. The patient and the family could relate strange signs and symptoms of mental illness. However, knowing the diagnosis without sufficient information could cause stress in the patient.

"When I discovered that I have a psychological problem, many problems were solved for me. The reason for my actions and thoughts was revealed to me." (Participant 8)

"My family thought I was addicted. When they find out about my disease, they've changed their behaviour with $m e$ ". (Participant 13)

"My psychiatrist told me the name of the disease and gave me some information about the symptoms and medications. It was really stressful to know you have a mental disorder". (Participant 5)

\section{Reaching a relative insight}

The second sub theme was "Reaching a relative insight". After disclosure of the diagnosis to patients with bipolar disorder, they were seeking information about their own illness. They obtained information from sources such as books, other patients, and the internet. They also received some information at the time of hospitalization or visit by their therapists. However, according to patients, their information was not sufficient to make informed decisions about the treatment process and important issues. In their opinion, the information they received from the treatment team was not enough.

"My psychiatrist told me that I have bipolar disorder. I did not get much information. My treatment was almost compulsory. I did not know if I had any other options besides taking medicine". (Participant 9)

"The psychiatrist asked me to consult him/her whenever I want to get married or make an important decision. I cannot make important decisions myself". (Participant 14)

"After finding out I have bipolar disorder, I started look- ing for information in books and surfing the Internet". (Participant 11)

\section{Discussion}

The purpose of this study was to investigate the patients with bipolar disorder experiences of disclosing their diagnosis. The results showed that patients are confused at the beginning of the disorder. They experience life in a new way that they had not experienced before. Ultimately patients are referred to psychiatrists or psychologists because of signs and symptoms of illness. But the confusion continues as long as the patient finds out something is wrong. When the patient finds out there is a problem, he or she is looking for the reason.

Usually, patients ask about their disease name when they are in the hospital or during a visit. Therapists usually disclose the name of the disease, but they do not give enough information to the patients. The disclosure of diagnosis without complementary information is like a limited brightness. Lack of enough information about the disease causes new problems for the patient. Patients gradually realize that they need to have more information to solve their problems. They seek to get more information about their disease and reach a relative insight into their disease.

Confusion is one of the most common emotions in the onset of psychiatric illnesses, including bipolar disorder (16). The symptoms like delusions and hallucinations make patients confused (22). Confusion among the unknowns is the source of stress for the patient and family. The patient becomes stigmatized for strange behaviors and thoughts (23). The family does not know how to deal with the patient. Patients with bipolar disorder have a higher prevalence of substance abuse $(24,25)$. They also may attempt or commit suicide (26). Since the patient and the family do not find any reason for these behaviors, they have a sense of hopelessness and frustration (27).

Our results showed that diagnosis disclosure usually occurs when patients are asked about their illness. This shows that psychiatrists do not have an active approach in the disclosure of the diagnosis to the patients (15). Non-disclosure of diagnosis is a common approach in medical models (19, 20, 28). Disclosure of the diagnosis without enough information can also cause problems. Previous research results showed that patients with bipolar disorder did not receive enough information about their illness $(29,30)$. Diagnosis disclosure without providing enough information is like limited lighting, which is helpful but not sufficiently informative. However, this limited brightness helps the patient to seek help and information.

In a systematic review, nine studies were found which were dealt with symptoms and diagnosis of patients with a bipolar diagnosis. These studies were conducted in Australia, the UK and the US. The analysis showed nine themes (31). Themes were struggles with identity, loss of control, disruption, uncertainty and instability, the negative impact of symptoms across life and the experience of loss, negative view of the self, positive or desirable aspects of mania, struggling with the meaning of diagnosis, stigma and acceptance and hope. The findings of the present study is in 
line with the mentioned piblications, especially with the indices acceptance and hope (31).

Disclosure of diagnosis to all patients, including those who have mental health problems, is an essential part of treatment and recovery. Knowing the diagnosis and informed decision are among the basic rights of all patients. Most of our participants did not receive their diagnosis at the first stages of their treatment and usually it happened very late. This means that they were deprived of their basic right to autonomy. From the moral point of view disclosure of diagnosis is a very controversial topic (8) especially in psychiatry. Based on the principle of respect for autonomy, every patient has the right to know his/her own disease name and have enough information about it. It is the prerequisite of making informed decisions. In the field of psychiatry, based on the principle of non-maleficence, in some cases disclosure of diagnosis can harm the process of treatment. In the same way, based on the principle of beneficence, disclosing the diagnosis does not equally benefit all patients. Disclosing the diagnosis without providing enough information to all patients is not in compliance with the principle of justice. The main limitation of this study was that it was conducted only on patients with bipolar disorder. While our aim was to understand the process which patients with bipolar disorder going through, doing the same researches on other mental health disorders can help to achieve better understanding. Another limitation was that our main source was the patients and we did not access to their family and therapists. Having different viewpoints can help to reach a better understanding. We suggest future studies on the disclosure of other mental disorders and participating in other sources of data.

\section{Conclusion}

The results showed that the process of diagnosis disclosure in patients with bipolar disorder does not enable them to make informed decisions. They reach a relative insight regarding their disorder. Their information is not enough to make informed decisions regarding their treatment and important aspects of their lives. In the case of patients with psychiatric disorders, the timing of disclosure, and the content which patient receive is crucial. Patients need to have detailed information about their disorder. It is the duty of therapists to provide the needed information.

\section{Acknowledgments}

The authors thank all participants who take part in this study and participate in interviews.

\section{Conflict of Interests}

The authors declare that they have no competing interests.

\section{References}

1. Vazmalaei HA, Jolfaei AG, Shabani A. Mood disorders insight scale: Validation of Persian version. J Res Med Sci. 2012;17(2):186-9.

2. Tully A, Murphy E, Smyth S, Conway Y, Geddes J, Devane D, et al. Interventions for the management of obesity in people with bipolar disorder. Cochrane Database Syst Rev. 2018;2018(4):CD013006.

3. Shabani A, Teimurinejad S, Kokar S, Ahmadzad Asl M, Shariati B,
Mousavi Behbahani Z, et al. Suicide Risk Factors in Iranian Patients With Bipolar Disorder: A 21- Month Follow-Up From BDPF Study. Iran J Psychiatry Behav Sci. 2013;7(1):16-23.

4. Joslyn C, Hawes DJ, Hunt C, Mitchell PB. Is age of onset associated with severity, prognosis, and clinical features in bipolar disorder? A meta-analytic review. Bipolar Disord. 2016;18(5):389-403.

5. Clemente AS, Santos WJD, Nicolato R, Firmo JOA. Stigma related to bipolar disorder in the perception of psychiatrists from Belo Horizonte, Minas Gerais State, Brazil. Cad Saude Publica. 2017;33(6):e00050016.

6. Abazari P, Taleghani F, Hematti S, Malekian A, Mokarian F, Hakimian SMR, et al. Breaking bad news protocol for cancer disclosure: an Iranian version. J Med Ethics Hist Me. 2017;10:13.

7. Sheykh-Talimi M, Shariati-Nasab S, Zare MK, Omani-Samani R. The right to information and their exceptions in medical practices in the Iranian legal system. J Med Ethics Hist Med. 2016;9:15.

8. Zahedi F. The challenge of truth telling across cultures: a case study. J Med Ethics Hist Med. 2011;4:11

9. Frese FJ, 3rd, Knight EL, Saks E. Recovery from schizophrenia: with views of psychiatrists, psychologists, and others diagnosed with this disorder. Schizophr Bull. 2009;35(2):370-80.

10. Anonymous. National Consensus Statement on Mental Health Recovery definition [Available from: http://mentalhealth. samhsa.gov/publications/allpubs/sma05-4129.

11. Corcoran J. The lived experience of people with bipolar disorder. Living with Mental Disorder: Routledge; 2016. p. 171-96.

12. Laugharne R, Priebe S. Trust, choice and power in mental health: a literature review. Soc Psychiatry Psychiatr Epidemiol. 2006;41(11):843-52.

13. Laugharne R, Priebe S, McCabe R, Garland N, Clifford D. Trust, choice and power in mental health care: experiences of patients with psychosis. Int J Soc Psychiatry. 2012;58(5):496-504.

14. Wild R, Pettit T. Conspiracy of silence? Telling patients with schizophrenia their diagnosis. Psychiatric Bullet. 2002;26(1):37-.

15. Bril-Barniv S, Moran GS, Naaman A, Roe D, Karnieli-Miller O. A Qualitative Study Examining Experiences and Dilemmas in Concealment and Disclosure of People Living With Serious Mental Illness. Qual Health Res. 2017;27(4):573-83.

16. Moran G, Mashiach-Eizenberg M, Roe D, Berman Y, Shalev A, Kaplan Z, et al. Investigating the anatomy of the helping relationship in the context of psychiatric rehabilitation: the relation between working alliance, providers' recovery competencies and personal recovery. Psychiatry Res. 2014;220(1-2):592-7.

17. Paul S, Nadkarni VV. A qualitative study on family acceptance, stigma and discrimination of persons with schizophrenia in an Indian metropolis. Int Soc Work. 2014;60(1):84-99.

18. Abe Y, de Kernier N, Oshima K. Partial disclosure of a co-diagnosis of bipolar disorder in a woman with borderline personality disorder. Innov Clin Neurosci. 2011;8(5):13-4.

19. Pandya A, Bresee C, Duckworth K, Gay K, Fitzpatrick M. Perceived impact of the disclosure of a schizophrenia diagnosis. Commun Ment Health J. 2011;47(6):613-21.

20. Seeman MV. When and how should I tell? Personal disclosure of a schizophrenia diagnosis in the context of intimate relationships. Psychiatr Q. 2013;84(1):93-102.

21. Braun V, Clarke V. Using thematic analysis in psychology. Qual Res Psychol. 2006;3(2):77-101.

22. Duppils GS, Wikblad K. Patients' experiences of being delirious. J Clin Nurs. 2007;16(5):810-8

23. Sean B. Guardedness in communications between people experiencing acute psychosis and mental health nurses. Dublin: City University; 2016.

24. Messer T, Lammers G, Muller-Siecheneder F, Schmidt RF, Latifi S. Substance abuse in patients with bipolar disorder: A systematic review and meta-analysis. Psychiatry Res. 2017;253:338-50.

25. Lanfredi M, Zoppei S, Ferrari C, Bonetto C, Van Bortel T, Thornicroft G, et al. Self-stigma as a mediator between social capital and empowerment among people with major depressive disorder in Europe: the ASPEN study. Eur Psychiatry. 2015;30(1):58-64.

26. Swartz-Vanetik M, Zeevin M, Barak Y. Scope and Characteristics of Suicide Attempts Among Manic Patients With Bipolar Disorder. Crisis. 2018;39(6):489-92.

27. September U, Beytell AM. Family Members' Experiences: People with Comorbid Bipolar and Substance Use Disorder. Int J Ment Health Addict. 2018.

28. Moran GS, Oz G, Karnieli-Miller O. Psychiatrists' challenges in 
Diagnosis disclosure in patients with bipolar disorder

considering disclosure of schizophrenia diagnosis in Israel. Qual Health Res. 2014;24(10):1368-80.

29. Bassett J, Lloyd C, Bassett H. Work Issues for Young People with Psychosis: Barriers to Employment. Br J Occup Ther. 2001;64(2):6672.

30. Thornicroft G, Rose D, Mehta N. Discrimination against people with mental illness: what can psychiatrists do? Adv Psychiatr Treat. 2010;16(1):53-9.

31. Russell L, Moss D. A Meta-Study of Qualitative Research Into the Experience of 'Symptoms' and 'Having a Diagnosis' for People Who Have Been Given a Diagnosis of Bipolar Disorder. Eur J Psychol. 2013;9(3):643-63. 